Check for updates

Cite this: RSC Adv., 2019, 9, 6118

Received 4th November 2018

Accepted 5th February 2019

DOI: $10.1039 / \mathrm{c} 8 \mathrm{ra0} 0130 \mathrm{~g}$

rsc.li/rsc-advances

\section{Silica cubosomes templated by a star polymer $\uparrow$}

\author{
Congcong Cui, (D) ${ }^{a}$ Lu Han (D) ${ }^{* a}$ and Shunai Che (D) ab
}

\section{Introduction}

Due to the versatile design of block copolymers (BCPs), the solution self-assembly of amphiphilic BCPs leads to a variety of materials with a well-defined morphology, and nanostructures with desirable functions are obtained due to the arrangement and physicochemical properties of the building blocks. ${ }^{1}$ The structure of the BCP system can be manipulated by the incompatibility and volume fractions of the different blocks and is associated with the packing parameter $g=V / a_{0} l$, where $V$ is the volume of the hydrophobic segment, $a_{0}$ is the molecular area of the hydrophilic head group, and $l$ represents the dynamic chain length of the hydrophobic part. ${ }^{2}$ By increasing the value of the packing parameter, spherical, cylindrical, bicontinuous, lamellar, and their corresponding inverted phases can be formed.

Among these materials, colloidal particles with bicontinuous structures (cubosomes) are the focus of an increasing number of studies due to their controllable structures and extensive applications. $^{3-8}$ These bicontinuous structures consist of two interwoven labyrinths separated by a single continuous surface that follows the triply periodic minimal surface. ${ }^{9}$ The attraction of these materials lies not only in their complex and highly symmetrical structures, but also in their structural analogies in both natural and artificial systems e.g. cubic membranes, ${ }^{10}$ the lyotropic liquid crystal phases of amphiphilic molecules, ${ }^{11,12}$ the self-assembly of block copolymers, ${ }^{13} \mathrm{etc}$.

The cubosomes show high water-dispersibility, chemical stability, and oral bioavailability. ${ }^{14-17}$ The interior of the

${ }^{a}$ School of Chemical Science and Engineering, Tongji University, 1239 Siping Road, Shanghai, 200092, China. E-mail: luhan@tongji.edu.cn

${ }^{b}$ School of Chemistry and Chemical Engineering, State Key Laboratory of Metal Matrix Composites, Shanghai Jiao Tong University, 800 Dongchuan Road, Shanghai, 200240, P. R. China

$\dagger$ Electronic supplementary information (ESI) available. See DOI: $10.1039 / \mathrm{c} 8 \mathrm{ra09130g}$ cubosomes consists of regular interpenetrating networks with a large surface area. Therefore, polymer cubosomes are widely used in the fields of drug delivery, catalysis and so on.$^{8,16-19}$ For the formation of polymer cubosomes, both top-down and bottom-up methods have been applied. The examples include self-organized precipitation by slow evaporation of a good solvent from a polymer solution containing both poor and good solvents, ${ }^{20-23}$ a spray drying technique for the synthesis of cubosomes from dry powder, ${ }^{24,25}$ flash nanoprecipitation, spontaneous emulsification, etc.; however, cubosomes primarily consist of self-assembled lipid molecules or amphiphilic block polymers; this limits their use under extreme conditions such as in strong acids and alkalis, at high temperatures and in complex solution environments. Moreover, several mesoporous silica microspheres have been reported, ${ }^{26-28}$ with pore sizes in the mesoporous range.

Note that an evaporation-induced self-assembly method has been employed in the synthesis of mesoporous solids. However, it is still difficult to control the structure and morphology of the ordered macroporous scaffolds with a large unit cell parameter due to the complex interactions between inorganic precursors and polymers, and bulk structures are often formed in this case. Herein, we report the synthesis via the self-assembly of a star polymer with an inorganic precursor in the evaporation process, and it has been demonstrated that this method can be utilized to form silica cubosomes with a spherical morphology and controllable bicontinuous structures. Our strategy was to use the microphase-templating route through the self-assembly of the amphiphilic star polymer poly(ethylene glycol)(polystyrene) ${ }_{2}$ (sGS2) with one hydrophilic block (PEG), two hydrophobic blocks (PS) and a silica source, tetraethyl orthosilicate (TEOS), in a mixture of tetrahydrofuran (THF) and water. At a large THF/water ratio, the hydrophobic PS block was present in the THF-rich phase; therefore, water was driven out of the hydrophobic phase and mainly appeared in the hydrophilic PEG region. ${ }^{29}$ With the evaporation of solvents and the hydrolysis and condensation of TEOS, the sample formed two 
interwoven diamond labyrinths through cooperative selfassembly. As THF evaporated, the packing parameter decreased, causing the self-assembled structure to transform from an inverted bicontinuous double-diamond to a lamellar structure and eventually to silica cubosomes (Scheme 1). Finally, the template molecules could be removed by calcination.

\section{Experimental}

\section{Materials}

Diethanolamine (99\%, Sinopharm Chemical Reagent Co., Ltd), p-toluenesulfonyl chloride (99\%, J\&K), triethylamine $(99.5 \%$, Macklin), dimethyl pyridine, (99\%, Transoole Reagent Co., Ltd), tetraethyl orthosilicate (TEOS, 98\%, J\&K), 2-bromoisobutyryl bromide (98\%, TCI), 1,1,4,7,7-pentamethyldiethylenetriamine

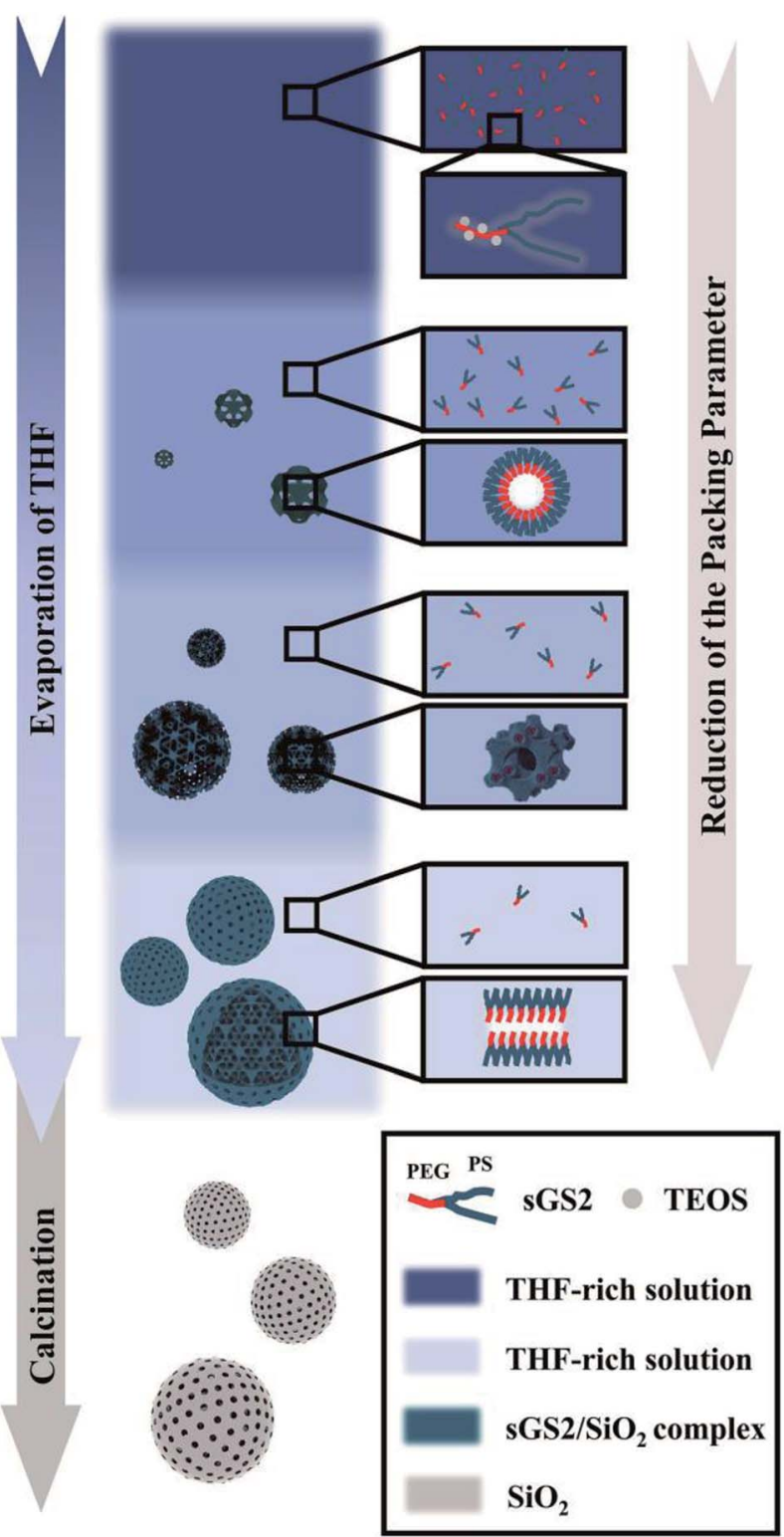

Scheme 1 Synthesis of silica cubosomes.
(PMEDTA, 98\%, TCI), copper bromide (CuBr, 97\%, Sinopharm Chemical Reagent Co., Ltd), stannous octoate ( $\mathrm{Sn}(\mathrm{Oct})_{2}, 95 \%$, Adamas-beta), diethyl ether (anhydrous) (99\%, Sinopharm Chemical Reagent Co. Ltd), hydrochloric acid ( $\mathrm{HCl}, 36 \%$, Sinopharm Chemical Reagent Co., Ltd), methanol (99\%, Sinopharm Chemical Reagent Co., Ltd), petroleum ether (60-90, Sinopharm Chemical Reagent Co., Ltd), N,N-dimethylformamide (DMF, 99.5\%, General-Reagent), dichloromethane (99.5\%, General-Reagent), tetrahydrofuran (THF, 99\%, Sinopharm Chemical Reagent Co., Ltd), $\varepsilon$-caprolactone (99\%, Adamasbeta), methylene blue trihydrate ( $82 \%$, General-Reagent), aminopropyltrimethoxysilane (APS, 96\%, TCI) and deionized water (Milli-Q, 18.2 M $\Omega \mathrm{cm}$ ) were used without further purification. The polymerization inhibitor of styrene (99\%, Sinopharm Chemical Reagent Co., Ltd) was removed by $20 \%$ sodium hydroxide solutions, dried with anhydrous magnesium sulfate, and finally distilled under reduced pressure before use. Methyl poly(ethylene glycol) with a hydroxyl terminal group (PEG-OH $5000)$ at one end was purchased from Aldrich $\left(M_{\mathrm{n}}, \mathrm{GPC}=5.17\right.$ $\mathrm{kg} \mathrm{mol}{ }^{-1}$, PDI $=1.12$, labelled as PEG). The DNA sodium salt from herring testes was purchased from Sigma, USA, listed as Type XIV and processed by the methods reported in our previous study. ${ }^{30}$

\section{Synthesis of PEG-OTs}

Poly(ethylene glycol) (20 g), which was previously dried in vacuo at $80{ }^{\circ} \mathrm{C}$ for $4 \mathrm{~h}$, was dissolved in methylene chloride $(55 \mathrm{~mL})$. Then, $p$-toluenesulfonyl chloride $\left(\begin{array}{ll}4 & \mathrm{~g}\end{array}\right)$ and triethylamine $(20 \mathrm{~mL})$ were added to this solution, and the mixture was stirred overnight under a nitrogen atmosphere. The reaction mixture was concentrated to about $30 \%$ of its original volume and then stirred for another $2 \mathrm{~h}$ at room temperature. The polymer was precipitated from this solution by the dropwise addition of ether $(300 \mathrm{~mL})$ under rapid stirring. Stirring was continued for another $15 \mathrm{~min}$, and the reaction mixture was placed in an ice bath. The precipitate was filtered, washed with ether, and dried in vacuo to obtain PEG-OTs.

\section{Synthesis of PEG-N-OH}

PEG-OTs (5 g), triethylamine $(6.95 \mathrm{~mL})$ and diethanolamine $(4 \mathrm{~mL})$ in DMF $(40 \mathrm{~mL})$ were heated at $80{ }^{\circ} \mathrm{C}$ for $12 \mathrm{~h}$. After cooling down the abovementioned solution to room temperature, the product was precipitated by the dropwise addition of ether to the solution. The precipitated PEG-N-OH $\mathrm{OH}_{2}$ was filtered, washed, and dried in vacuo.

\section{Synthesis of PEG-N- $\mathrm{Br}_{2}$}

PEG-N-OH $(4 \mathrm{~g})$ was dissolved in dry tetrahydrofuran $(20 \mathrm{~mL})$. Then, $4 \mathrm{~mL}$ of triethylamine was added to the abovementioned solution. After the solution was stirred for $30 \mathrm{~min}$ while being cooled down in an ice-water bath, 2-bromoisobutyryl bromide $(0.99 \mathrm{~mL})$ was added to the solution over $1 \mathrm{~h}$ under $\mathrm{N}_{2}$ protection. Finally, the solution was stirred at room temperature for $12 \mathrm{~h}$ and then filtered to obtain a homogeneous solution. Subsequently, $500 \mathrm{~mL}$ of cold ether was added to the abovementioned solution to obtain a white precipitate. The 
precipitate was filtered and washed several times with cold ether. After the precipitate was dried under vacuum, the macroinitiator $\mathrm{PEG}-\mathrm{N}-\mathrm{Br}_{2}$ was obtained.

\section{Synthesis of PEG-PS 2 by atom transfer radical polymerization (ATRP)}

The PEG-PS ${ }_{2}$ star polymers were synthesized using the wellestablished ATRP of styrene at $110{ }^{\circ} \mathrm{C}$ with PEG-N- $\mathrm{Br}_{2}$ as the macroinitiator and CuBr/PMDETA as the catalyst. Typically, PEG-N-Br ${ }_{2}(1.00 \mathrm{~g})$ was dissolved in styrene $(10.00 \mathrm{~g})$ in a $100 \mathrm{~mL}$ Schlenk flask, and then, PMDETA $(0.17 \mathrm{~mL})$ and $\mathrm{CuBr}$ $(0.12 \mathrm{~g})$ were added to the abovementioned solution. The reaction system was fully degassed via more than three freezepump-thaw cycles and sealed under vacuum. The bottle was then placed in an oil bath at $110{ }^{\circ} \mathrm{C}$ to allow the polymerization to occur. After the polymerization was terminated by exposing the reaction mixture to air, the catalyst was removed by filtration through an $\mathrm{Al}_{2} \mathrm{O}_{3}$ column using methylene chloride as the eluent. Cold methanol $(500 \mathrm{~mL})$ was poured into the clear

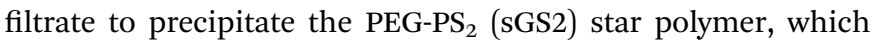
was obtained by filtration and dried under vacuum at room temperature.

\section{Synthesis of macroporous silica}

The macroporous silica was prepared via solvent-evaporationinduced aggregation assembly, and the miktoarm star polymer was used as the template. Then, $0.10 \mathrm{~g}$ of sGS2 was added to $7.00 \mathrm{~g}$ of THF with $5.00 \mathrm{~g}$ of HCL $(2 \mathrm{M})$ for the hydrolysis of TEOS. After the mixture was stirred for approximately $2 \mathrm{~h}$ at $50{ }^{\circ} \mathrm{C}, 0.70 \mathrm{~g}$ of TEOS was finally added to the solution, and then, the mixture was allowed to stir for an additional $2 \mathrm{~h}$. The solvent was then allowed to completely evaporate at $50{ }^{\circ} \mathrm{C}$. The as-made samples were calcined at $550^{\circ} \mathrm{C}$ in air for $6 \mathrm{~h}$ to remove the template.

\section{Surface amination of the silica cubosomes}

Typically, $0.125 \mathrm{mg}$ silica cubosomes were added to $12.5 \mathrm{~mL}$ of $10 \%$ (vol\%) aminopropyltrimethoxysilane in toluene. The solution was reacted at $80{ }^{\circ} \mathrm{C}$ for $12 \mathrm{~h}$. The aminated silica cubosomes were then centrifuged and washed with water and ethanol.

\section{Adsorption experiment}

Herein, $20 \mathrm{mg}$ silica cubosomes were added to $20 \mathrm{~mL}$ of a $10 \mathrm{mg} \mathrm{\textrm {L } ^ { - 1 }}$ methylene blue solution. Alternatively, $20 \mathrm{mg}$ aminated silica cubosomes were added to $20 \mathrm{~mL}$ of a $20 \mathrm{mg} \mathrm{L}^{-1}$ DNA solution. After a certain period of time, $3 \mathrm{~mL}$ of the solution was taken out, and the adsorbent was centrifuged. The supernatant was analyzed by UV-visible absorption spectroscopy.

\section{Characterizations}

The nuclear magnetic resonance (NMR) spectra were obtained via the Bruker Avance $600 \mathrm{MHz}$ NMR spectrometer using tetramethylsilane (TMS) as the internal reference. The polymers were dissolved in deuterated chloroform $\left(\mathrm{CDCl}_{3}\right)$. The molecular weights and molecular weight distributions of the polymers were determined via the HLC-8320GPC (TOSOH Corp.) gel permeation chromatography (GPC) apparatus, and the measurements were conducted using DMF as the eluent at $40{ }^{\circ} \mathrm{C}$ at the elution rate of $0.6 \mathrm{~mL} \mathrm{~min}{ }^{-1}$. The apparent molecular weights were determined via the Tskgel AWM-H $(6.0 \mathrm{ID} \times 150 \mathrm{~mm})$ columns using linear polystyrene standards. The SAXS experiments were conducted by beamline BL16B1 at the Shanghai Synchrotron Radiation Facility. The microscopic features of the samples were observed using SEM, which was performed using JEOL JSM-7401F, JSM-7800F Prime and JSM-7900F; the samples were observed with a landing energy of $1 \mathrm{kV}$ without any metal coating. TEM observations were performed using the JEOL JEM-2100 microscope equipped with a LaB6 gun operated at $200 \mathrm{kV}(\mathrm{Cs}=1.0 \mathrm{~mm}$, point resolution $2.3 \AA$ ). Images were obtained using the TENGRA CCD camera (with a resolution of $2304 \times 2304$ pixels, a $2: 1$ fibreoptical taper and an effective pixel size of $8 \mu \mathrm{m}^{2}$ ). The zeta potential was obtained using Malvern Zetasizer Nano ZS90. UVVis spectra were obtained by Persee TU-1810. Finally, the nitrogen adsorption-desorption experiment was performed using Quantachrome Instruments Autosorb iQ.

\section{Results and discussion}

\section{Properties of the miktoarm star polymer (sGS2)}

We simulated the structure of the phospholipid molecules to synthesize a star polymer with one hydrophilic block and two hydrophobic blocks (Fig. S1†). The amphiphilic star polymer can cause microphase separation due to the incompatibility between two blocks in the solution; therefore, it undergoes selfassembly to form a variety of complex structures.

The $M_{\mathrm{n}}$ of sGS2 star polymers is $51.40 \mathrm{~kg} \mathrm{~mol}^{-1}$ with a volume fraction of $\mathbf{1 0 . 1 9 \%}$ for the PEG segment (see Table 1). The Flory-Huggins interaction parameter for the two blocks has been calculated as 139.02 by

$$
\chi_{\mathrm{N}}=\sum N \times \frac{V_{\mathrm{ref}}\left(\delta_{\mathrm{PS}}-\delta_{\mathrm{PEG}}\right)^{2}}{R T}
$$

where $V_{\text {ref }}$ is the segment reference volume $\left(V_{\text {ref }}=100 \mathrm{~cm}^{3}\right.$ $\mathrm{mol}^{-1}$ ) and $\delta$ is the Hildebrand solubility parameter of the polymer $\left(\delta_{\mathrm{PS}}=19.07\left(\mathrm{~J} \mathrm{~cm}^{-3}\right)^{1 / 2}\right.$ and $\left.\delta_{\mathrm{PEG}}=21.55\left(\mathrm{~J} \mathrm{~cm}^{-3}\right)^{1 / 2}\right)$. The Flory-Huggins interaction parameter $\chi_{\mathrm{N}} \gg 10.5$ indicates the tendency of the star polymer to form a strong microphase between the PEG and PS blocks.

The nuclear magnetic resonance (NMR) spectra of the polymers are shown in Fig. 1. The polymers were dissolved in deuterated chloroform. The resonance signals of the methoxy protons and PEG backbone protons appear at 3.40 and 3.80$3.50 \mathrm{ppm}$, respectively. The resonance signals of the PS backbone protons and benzene ring protons appear at 1.98-1.12 and 7.23-6.26 ppm, respectively, which confirm the presence of PS. Moreover, the $a / l$ signal ratio is $3: 2$, which proves that the star polymers have one PEG backbone and two PS backbones.

The $M_{\mathrm{n}}(\mathrm{NMR})$ is calculated by the degree of polymerization (DP). ${ }^{31,32}$ The ratio of the peak areas between 3.80 and $3.50 \mathrm{ppm}$ and 7.23 and $6.26 \mathrm{ppm}$ is 4.77 . Therefore, the total DP for the PS 
Table 1 Characterizations of the polymers

\begin{tabular}{llllr}
\hline Polymers & $M_{\mathrm{n}}(\mathrm{NMR})\left(\mathrm{kg} \mathrm{mol}^{-1}\right)$ & $M_{\mathrm{n}}(\mathrm{GPC})\left(\mathrm{kg} \mathrm{mol}^{-1}\right)$ & PDI & $V_{\mathrm{PEG}} \%$ \\
\hline SGS2 & 51.40 & 40.00 & 1.84 & 10.19
\end{tabular}

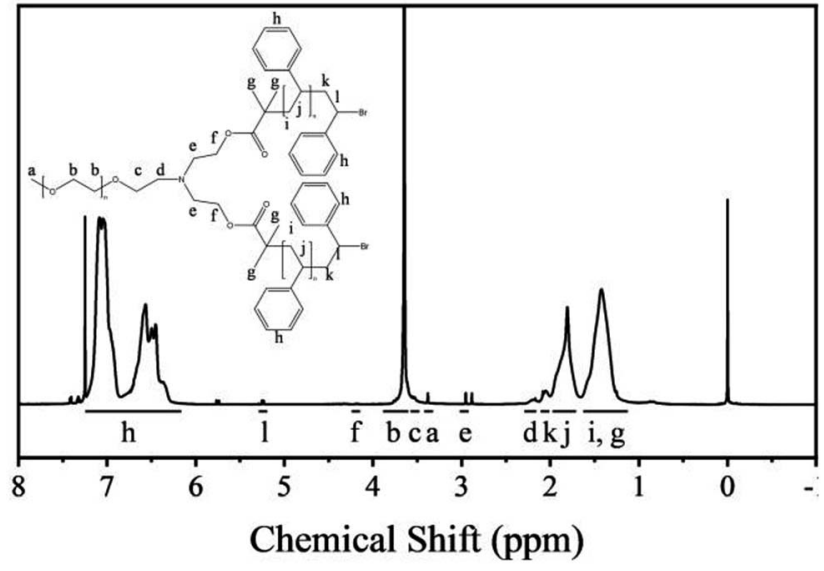

Fig. $1{ }^{1} \mathrm{H}$ NMR spectra of the polymers.

block is 446. This can be used as a basis for estimating the $M_{\mathrm{n}}(\mathrm{NMR})$ (that is, $M_{\mathrm{n}}(\mathrm{NMR})=M_{\mathrm{PEG}}+\mathrm{DP} \times M_{\text {monomer }}$ ) of star polymers, and the $M_{\mathrm{n}}(\mathrm{NMR})$ has been estimated to be $51.40 \mathrm{~kg}$ $\mathrm{mol}^{-1}$

\section{Characterization of the cubosome structures}

Macroporous silica was synthesized by evaporation-induced self-assembly with sGS2 as the templating agent. As the evaporation process proceeds, the PEG and PS blocks experience microphase separation and thus form different structures in the solution. Moreover, TEOS gradually hydrolyzes and bonds with PEG through hydrogen bonding interactions. After the completion of evaporation, the organic templating agents were removed by calcination to obtain silica with an ordered structure.

Fig. 2a shows the low-magnification scanning electron microscopy (SEM) image of the sample, presenting a spherical morphology with 2-15 $\mu \mathrm{m}$ diameter. The surfaces of these spherical particles show ordered pores (Fig. $2 \mathrm{~b}$ ) with hexagonal perforation and $\sim 70 \mathrm{~nm}$ diameter, which are formed by the change in structure from bicontinuous to lamellar (Scheme 1). After crushing the particles into pieces, the inner structure can be revealed. As shown in Fig. 2c, the cross sections of the particles show that the silica frameworks contain smooth surfaces with tetrahedral arrangements of the nodes, which can be identified as a diamond structure. The structure consists of two sets of diamond networks with an inner diameter of $\sim 26 \mathrm{~nm}$ and a wall thickness of $\sim 18 \mathrm{~nm}$. The unit cell parameter obtained from the SEM image is $a \approx 250 \mathrm{~nm}$ with the original cubic symmetry (space group $P n \overline{3} m$ ). Note that one channel of the diamond networks is connected to the hexagonal array of openings on the surface of the spheres (Fig. 2d). The TEM images (Fig. 2e and S3†) of the thin section of the sample after slicing show ordered hollow network structures with the contrast of the double diamond structure. Note that the structure transforms into a layered structure approaching the surface. However, due to the extremely large unit cell parameter, it is very difficult to measure the small angle X-ray scattering profile of this sample because the first reflection exceeds the limit of the instrument (Fig. 2f).

The nitrogen adsorption-desorption isotherm of the silica cubosomes exhibits a complex behaviour due to the hierarchical porous structure (Fig. S4a†). It shows a type II isotherm with an $\mathrm{H} 4$ type hysteresis loop, and a sharp increase in the relative pressure range of $0.90-0.99$ indicates the presence of macropores. The surface area is calculated as $693 \mathrm{~m}^{2} \mathrm{~g}^{-1}$ by the NLDFT method. The relatively high surface area is mainly contributed by the mesopores. The pore size distribution obtained from the NLDFT method is broad (Fig. S4b†). This is
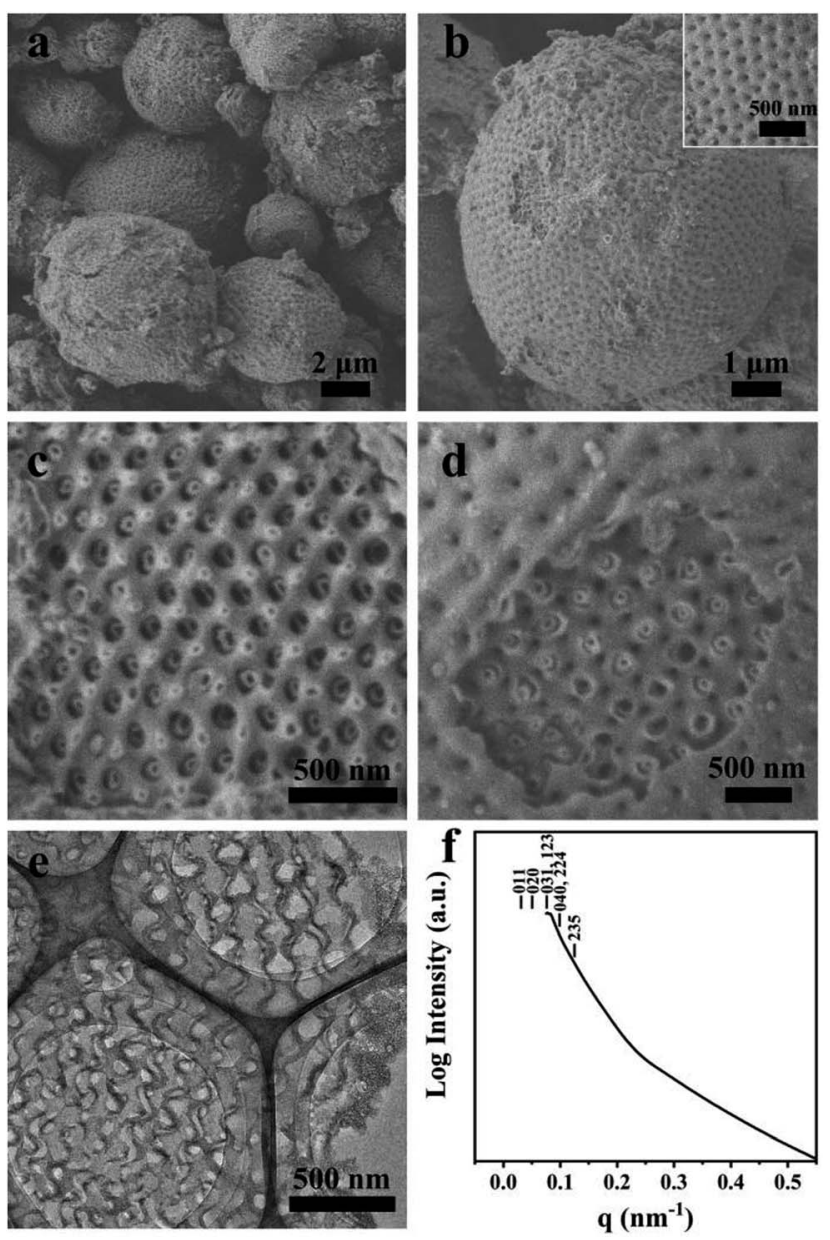

Fig. 2 SEM (a-d), TEM (e) images and SAXS pattern ( $f$ ) of the synthesized sample. The mass ratio of sGS2: THF : $\mathrm{HCl}(2 \mathrm{M}):$ TEOS is $1: 70: 50: 7$. 
mainly due to the existence of multi-level pores, whereas the large macropores cannot be accurately predicted.

The surface potentials of the as-made sample and the calcined sample in the aqueous medium are $-18.87 \mathrm{mV}$ and $-11.18 \mathrm{mV}$, respectively. The as-made sample can stably disperse in THF for a long time and in water for 12 hours (Fig. S5 $\dagger$ ). However, the calcined sample in the aqueous medium precipitates within 3 hours. This is due to the fact that the as-made sample consists of silica coated with a large number of stretched PS blocks (the PEG block is embedded inside the silica). Therefore, it can stably disperse in THF; however, it cannot stably disperse in an aqueous medium for a long time. The calcined sample is completely composed of silica, and the $\mathrm{Si}-\mathrm{OH}$ groups have been removed during the calcination step; therefore, the sample cannot form a stable dispersion in an aqueous medium. However, this dispersity can be improved by further surface modification.

Moreover, the adsorption of silica cubosomes on methylene blue and DNA was explored (Fig. S7†). The maximum adsorption ratio for methylene blue was $79.0 \%$ for 2 hours. Since the silicon hydroxy group on the surface of the silica has a negative charge, it has no adsorption effect on the negatively charged DNA molecules. By aminating the surface of silica cubosomes, the silicon hydroxy group can adsorb DNA molecules in a neutral aqueous medium. The relationship between the adsorption rate and time was calculated by measuring the absorbance change at $258 \mathrm{~nm}$, which reached a maximum of $95.2 \%$ at $90 \mathrm{~min}$. The adsorption of small molecules on porous materials is mainly related to the surface area of these materials. As the surface area of silica cubosomes is not advantageous as compared to that of mesoporous materials, their adsorption on methylene blue is limited. However, the large pore size and accessibility of the porous structure play an important role for the diffusion and adsorption of macromolecules into the material.

\section{Formation mechanism of cubosomes}

To further understand the formation mechanism of cubosomes, a series of samples with an ordered structure have been synthesized by changing the amounts of THF, $\mathrm{HCl}$ and the template. The three-component synthesis-field diagram based on the mass fraction of THF, $\mathrm{HCl}(2 \mathrm{M})$ and sGS2 $\times 100$ (the mass of sGS2 in the diagram is 100 times that of the actual quantity) is shown in Fig. 3. The three-component synthesisfield diagram drawn without the 100 times relationship is shown in Fig. S6. $\dagger$ Inverse 2D hexagonal, bulk double diamond, cubosomes and disordered structures have been obtained.

Fig. 4 presents the SEM images of the calcined sample with an inverse $2 \mathrm{D}$ hexagonal synthesized with a mass ratio of sGS2 : THF : $\mathrm{HCl}(2 \mathrm{M})=1: 120: 50$. The sample consisted of stacked tubes with an average diameter of $\sim 20 \mathrm{~nm}$ and a wall thickness of $\sim 5 \mathrm{~nm}$. The length ranges from several to tens of microns.

The bulk double diamond (Fig. 5) can be synthesized with a mass ratio of sGS2 : THF $: \mathrm{HCl}(2 \mathrm{M})=1: 80: 20$. The structure is similar to the shifted double-diamond structure reported in our previous study. ${ }^{29}$ Moreover, two reflections can be

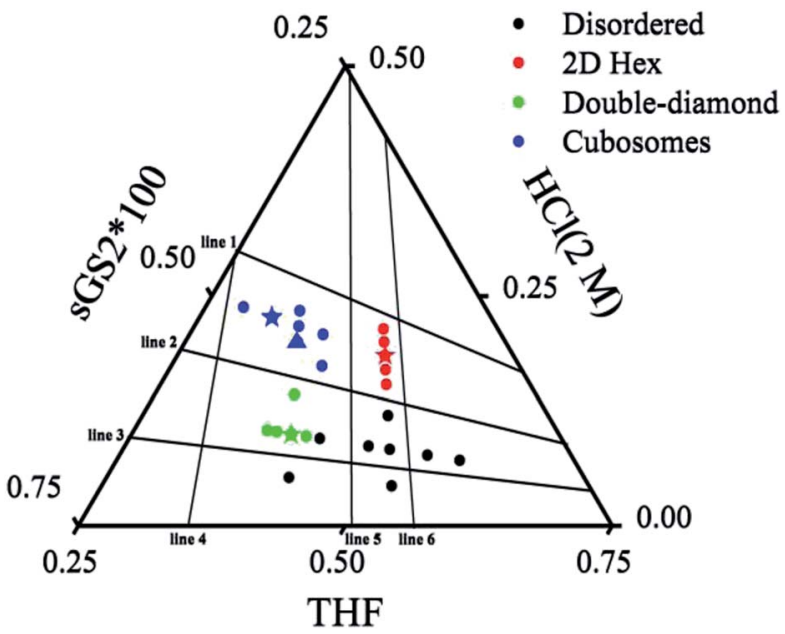

Fig. 3 Three-component synthesis-field diagram with structures derived from various compositions (in mass fractions) of the sGS2 polymer. The mass of sGS2 in the diagram is 100 times that of the actual quantity. The blue, red, green stars and blue triangle are the samples shown in Fig. 2, 4, 5, and 6, respectively.
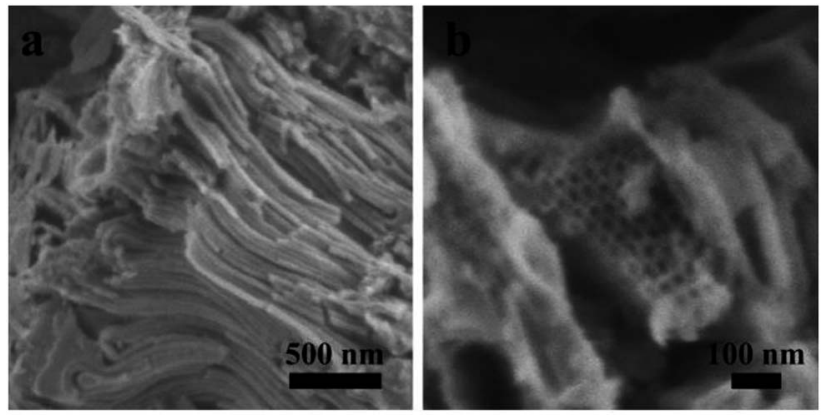

Fig. 4 SEM ( $a$ and b) images of the inverse 2D hexagonal structure.

observed in the SAXS pattern, which can be indexed to 101 and 112 (200) reflections with the unit cell parameters of $a \approx 54 \mathrm{~nm}$ and $c \approx 77 \mathrm{~nm}$ based on the tetragonal lattice. From the SEM images, two sets of silica frameworks can be observed, which show a tetrahedral connection at the nodes and can be considered as a shifted double diamond structure.

To illustrate the formation of different structures, several lines originating from zero points divided the diagram into several regions, which indicated different mass fractions of THF, $\mathrm{HCl}(2 \mathrm{M})$ and sGS $2 \times 100$, respectively. The three lines (line 1-3) derived from the point of THF indicate that with a change in the mass fraction of THF, $m_{\mathrm{HCl}(2 \mathrm{M})} / m_{\mathrm{SGS} 2 \times 100}$ remains fixed. Among them, line 2 separates hexagonal and cubosomes from double-diamond in two areas. In addition, three lines (line 4-6) derived from the point of $\mathrm{HCl}(2 \mathrm{M}$ ) indicate that $m_{\mathrm{THF}} / m_{\mathrm{SGS} 2 \times 100}$ remains fixed as the mass fraction of $\mathrm{HCl}$ ( $2 \mathrm{M}$ ) changes. Cubosomes and bulk double-diamond are formed in the area between line 4 and line 5 . As the mass fraction of $\mathrm{HCl}(2 \mathrm{M})$ increases, the structure of the resulting silica is transformed from bulk double-diamond to doublediamond structure with a perforated layered coverage and then is completely converted to cubosomes. 

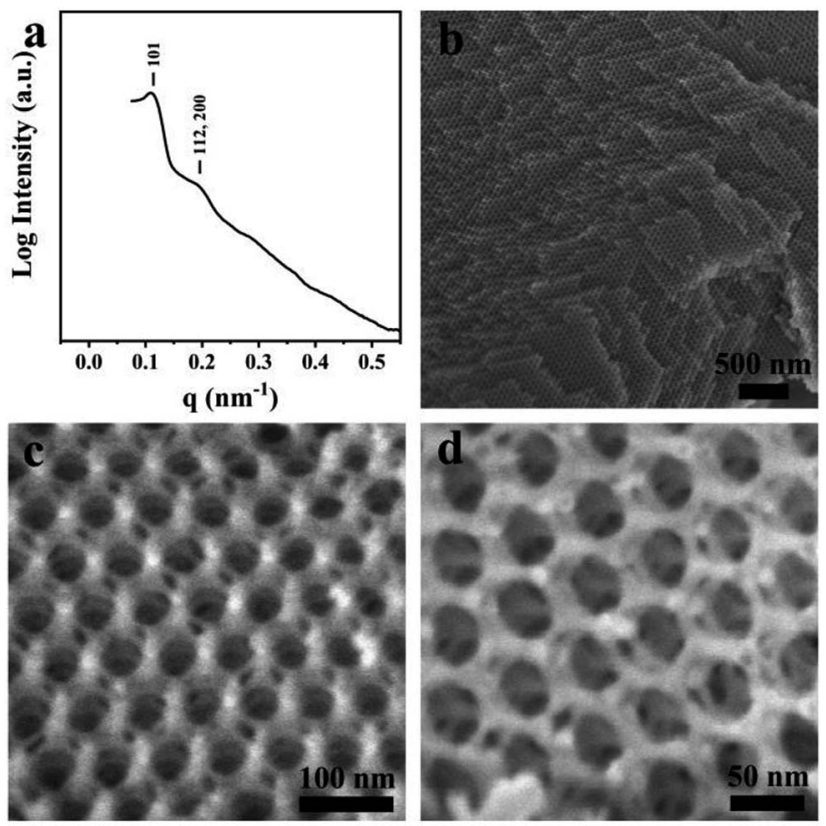

Fig. 5 SAXS (a) pattern and SEM (b-d) images of the double-diamond structure.

Based on the abovementioned results, the structural change from a bulk double-diamond structure to the cubosomes can be explained by the decrease in the THF content. Because the star polymer sGS2 has two hydrophobic tails that are similar to those of phospholipid molecules, it is easier to form bicontinuous structures. At the beginning of the reaction, the corporative self-assembly of the template molecules with an inorganic source leads to the formation of a nucleus with a doublediamond structure. Upon solvent evaporation, the structure gradually grows with the self organization of the template and the hydrolysis and condensation of TEOS. Due to the faster evaporation of THF, the packing parameters are reduced, leading to a decrease in the hydrophobic volume fraction. Therefore, the packing parameters are decreased, and the structure is changed from an inverted bicontinuous doublediamond to a lamellar structure. The double-diamond core is covered by layers of lamellar surface, and the cubosomes can be obtained. This process can be demonstrated by cubosomes with multiple layers of lamellar on the surface (Fig. 6). Moreover, the

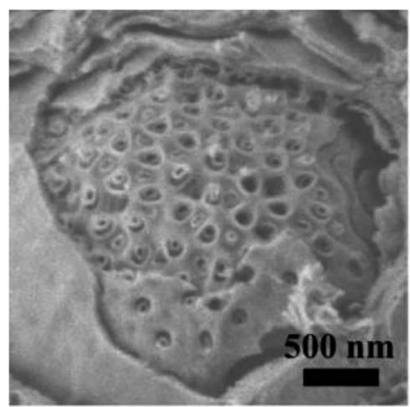

Fig. 6 An SEM image of the cubosomes with multiple layer lamellar on the surface. The sample was synthesized with a mass ratio of sGS2 : THF : $\mathrm{HCl}(2 \mathrm{M}):$ TEOS $=1: 80: 45: 7$. water content for the synthesis of cubosomes is relatively high; this leads to significantly enlarged unit cell parameters due to the expanded channels in the diamond framework. ${ }^{29}$ When the water content in the system is very high, the polymer will precipitate out of the solution due to the large hydrophobic tails of the star polymer. Therefore, it is difficult to form a normal micelle structure (oil-in-water) in our system.

\section{Conclusions}

We have showed the synthesis of silica cubosomes using an amphiphilic star polymer as a template, TEOS as a silicon source, and a volatile self-assembly method for preparing microporous silica in a THF and water system. Compared with mesoporous materials, our materials have larger pore diameters, which facilitate the diffusion of macromolecules inside the materials, and have high mass transfer efficiency. Due to the inorganic skeleton, the materials show enhanced stability. We hope that our strategy will provide a deeper understanding of the block copolymer systems and widen the synthesis and applications of new porous materials.

\section{Conflicts of interest}

There are no conflicts to declare.

\section{Acknowledgements}

This work was supported by the National Natural Science Foundation of China, (21533002, 21471099, 21571128), the National Key R\&D Program of China (2016YFC0205900), the National Excellent Doctoral Dissertation of PR China (201454), and the Shanghai Rising-Star Program (17QA1401700).

\section{Notes and references}

1 Y. Mai and A. Eisenberg, Chem. Soc. Rev., 2012, 41, 59695985.

2 J. N. Israelachvili, D. J. Mitchell and B. W. Ninham, J. Chem. Soc., Faraday Trans. 2, 1976, 72, 1525-1568.

3 Z. Lin, S. Liu, W. Mao, H. Tian, N. Wang, N. Zhang, F. Tian, L. Han, X. Feng and Y. Mai, Angew. Chem., Int. Ed., 2017, 56, 7135-7140.

4 M. G. Jeong and K. T. Kim, Macromolecules, 2016, 50, 223234.

5 Y. La, C. Park, T. J. Shin, S. H. Joo, S. Kang and K. T. Kim, Nat. Chem., 2014, 6, 534-541.

6 Q. Xiao, Z. Wang, D. Williams, P. Leowanawat, M. Peterca, S. E. Sherman, S. Zhang, D. A. Hammer, P. A. Heiney, S. R. King, D. M. Markovitz, S. André, H.-J. Gabius, M. L. Klein and V. Percec, ACS Cent. Sci., 2016, 2, 943-953.

7 C. Park, Y. La, T. H. An, H. Y. Jeong, S. Kang, S. H. Joo, H. Ahn, T. J. Shin and K. T. Kim, Nat. Commun., 2015, 6, 6392.

8 S. D. Aindrilla, M. C. Harsiddhi, R. J. Kisan and J. K. Vilasrao, Curr. Drug Delivery, 2016, 13, 482-493. 
9 S. Hyde, Z. Blum, T. Landh, S. Lidin, B. Ninham, S. Andersson and K. Larsson, The language of shape: the role of curvature in condensed matter: physics, chemistry and biology, Elsevier, 1996.

10 Y. Deng and M. Mieczkowski, Protoplasma, 1998, 203, 16-25. 11 V. Luzzati and P. A. Spegt, Nature, 1967, 215, 701.

12 V. Luzzati, A. Tardieu and T. Gulik-Krzywicki, Nature, 1968, 217, 1028.

13 L. Han and S. Che, Adv. Mater., 2018, 30, 1705708.

14 D. J. McClements, Adv. Colloid Interface Sci., 2015, 219, 2753.

15 E. Elisabetta, D. Markus, N. Claudio and C. Rita, Curr. Pharm. Des., 2016, 22, 5382-5399.

16 Z. Karami and M. Hamidi, Drug Discovery Today, 2016, 21, 789-801.

17 J. Mo, G. Milleret and M. Nagaraj, Liq. Cryst. Rev., 2017, 5, 69-85.

18 A. Zabara and R. Mezzenga, J. Controlled Release, 2014, 188, 31-43.

19 I. D. M. Azmi, S. M. Moghimi and A. Yaghmur, Ther. Delivery, 2015, 6, 1347-1364.

20 H. Yabu, T. Higuchi and M. Shimomura, Adv. Mater., 2005, 17, 2062-2065.
21 T. Higuchi, A. Tajima, H. Yabu and M. Shimomura, Soft Matter, 2008, 4, 1302-1305.

22 H. Yabu, Polym. J., 2012, 45, 261.

23 K. H. Ku, J. M. Shin, D. Klinger, S. G. Jang, R. C. Hayward, C. J. Hawker and B. J. Kim, ACS Nano, 2016, 10, 5243-5251.

24 M. H. Shah, S. V. Biradar and A. R. Paradkar, Int. J. Pharm., 2006, 323, 18-26.

25 P. T. Spicer, W. B. Small, M. L. Lynch and J. L. Burns, J. Nanopart. Res., 2002, 4, 297-311.

26 A. Chen, Y. Yu, H. Lv, Y. Zhang, T. Xing and Y. Yu, Mater. Lett., 2014, 135, 43-46.

27 R. Guillet-Nicolas, J.-L. Bridot, Y. Seo, M.-A. Fortin and F. Kleitz, Adv. Funct. Mater., 2011, 21, 4653-4662.

28 R. Longloilert, T. Chaisuwan, A. Luengnaruemitchai and S. Wongkasemjit, J. Sol-Gel Sci. Technol., 2011, 58, 427-435.

29 L. Han, D. Xu, Y. Liu, T. Ohsuna, Y. Yao, C. Jiang, Y. Mai, Y. Cao, Y. Duan and S. Che, Chem. Mater., 2014, 26, 70207028.

30 Y. Cao, K. Kao, C. Mou, L. Han and S. Che, Angew. Chem., Int. Ed., 2016, 55, 2037-2041.

31 C. Lv, C. He and X. Pan, Angew. Chem., Int. Ed., 2018, 57, 9430-9433.

32 H. Li, D. Yang, Y. Gao, H. Li and J. Xu, RSC Adv., 2015, 5, 96377-96386. 